\title{
Thrombolytic Stroke Treatment of a 12-Year-Old Girl with Intracranial Fibromuscular Dysplasia
}

\author{
Manuel Bolognese Martin Griebe Alex Foerster \\ Michael G. Hennerici Marc Fatar \\ Department of Neurology, Universitätsmedizin Mannheim, University of \\ Heidelberg, Mannheim, Germany
}

\section{Key Words}

Fibromuscular dysplasia $\cdot$ Pediatric stroke $\cdot$ Thrombolysis

\begin{abstract}
Fibromuscular dysplasia, predominantly found in adult women, is a rare disease of small and middle-sized arteries of the kidney and brain. We present a case of a 12-year-old girl with acute ischemic stroke, due to fibromuscular dysplasia of the distal internal carotid artery and the proximal middle cerebral artery, which was successfully treated with t-PA.
\end{abstract}

\section{Introduction}

Fibromuscular dysplasia (FMD) is a rare disease affecting small and middle-sized arteries of the kidney and brain, predominantly in adult women. It rarely occurs in children and has not previously been treated with thrombolytics following acute stroke.

\section{Case Report}

A 12-year-old girl was admitted to the pediatric emergency room after having collapsed during a soccer game. Family members reported that she had fallen down with convulsions of all four extremities. On admission, a neurological examination revealed a severe left-hemispheric syndrome with global aphasia, conjugated gaze deviation to the left, severe hemiparesis with extensor plantar response and hemihypesthesia of the right side [National Institute of Health Stroke Score (NIHSS): 19]. Both the family history and her medical history were unremarkable apart from a migraine without aura.

Acute cerebral MRI showed an acute middle cerebral artery (MCA) stroke on diffusion-weighted images involving the insula, the lentiform nucleus, the head of the nucleus caudatus and the centrum 
semiovale (fig. 1a), with occlusion of distal M3 branches of the left MCA and irregular contrasting of both the proximal left M1 and the distal left internal carotid artery (ICA) segments (fig. 1d).

Furthermore, perfusion-weighted images (fig. 1b) revealed an extended perfusion deficit beyond the territory of the diffusion-weighted image lesions in the cortical areas of the complete MCA territory, characteristic of a significant mismatch between the hypoperfused area and the diffusion impairment. The patient was treated within an extended time window of $5 \mathrm{~h}$ after onset of the symptoms with intravenous t-PA $(0.9 \mathrm{mg} / \mathrm{kg}$ body weight, resulting in $5 \mathrm{mg}$ as a bolus and $50 \mathrm{mg}$ continuously over 60 min). Forty-eight hours later, marginal improvement was observed (NIHSS after thrombolysis: 15). Laboratory studies were unremarkable as were an extended coagulation profile, vasculitis screening and analysis of the cerebrospinal fluid. Transthoracic and transesophageal echocardiography showed no abnormalities, while both extracranial and transcranial duplex sonography demonstrated a severe stenosis in the siphon of the left ICA with significantly decreased prestenotic flow velocity. Furthermore, the sonography results showed a retrograde flow direction in the left proximal anterior cerebral artery, indicating hemodynamic compensation from right to left anterior circulation. The size of the ischemia on a follow-up MRI the next day was unchanged and without secondary hemorrhagic transformation. On MR angiography, all 3 MCA segments, including the distal M3 branches, were recanalized while mild pathology within the proximal M1 and the distal ICA persisted. Digital subtraction angiography of these vessel segments showed typical constrictions with 'strings of beads' characteristic of FMD (fig. 1c).

We treated the patient with acetylsalicylic acid $100 \mathrm{mg}$, physiotherapy and speech therapy. Another follow-up MRI at day 16 showed a new - though clinically asymptomatic - ischemic lesion in the left MCA territory, which prompted oral anticoagulant treatment with phenprocoumon (INR 2.0-3.0). The patient was released into rehabilitation after further improvements (NIHSS on discharge: 8 ) and presented with severe paresis of the right arm and moderate paresis of the right leg. Three months later we saw her again with further clinical improvement (NIHSS: 5): only moderate right spastic hemiparesis and mild residual aphasia persisted. She was able to walk without any help. Neuroimaging and vascular studies were unchanged.

\section{Discussion}

Stroke in children is rare, with an incidence varying from 1.3 to 13.0 per 100,000 [1]. While systemic thrombolysis with t-PA is considered the gold standard of acute treatment in adult ischemic stroke patients [2,3], insufficient data on systemic intravenous or local intra-arterial thrombolysis in children are available [4-6]. A major problem is the delay in diagnosis of stroke, which is even more challenging than in adults, as this diagnosis is rarely assumed by parents, family members or doctors [7]. Although stroke in young adults is often due to similar causes as those in the elderly, active mechanisms in children are different.

FMD is very rare in children and has only been reported in a few casual communications [8-10]. As MR angiography is unable to provide sufficient resolution to demonstrate small multisegmental structural changes of commonly multiple brainsupplying arteries, digital subtraction angiography is needed [11]. Therefore, FMD of the carotid or intracranial arteries often remains undiagnosed. Treatment and prevention concepts are not evidence-based. The use of platelet inhibitors or oral anticoagulation for 6-12 months after clinical events in adults is a possible regimen in children as well [12].

As a differential diagnosis, transient cerebral angiopathy (TCA) was discussed. Despite being frequently considered as a reason for stroke in children with focal stenosis of the intracranial vessels, the diagnosis of TCA does not describe a particular underlying pathophysiology and, other than an association with varicella zoster infections, there is little known about its etiology [13]. An overlap of TCA with FMD cannot be excluded. 
To our knowledge, this is the first report of a child with FMD treated with t-PA, and also the first one that describes thrombolysis of an acute stroke which was based on MRI diffusion/perfusion mismatch criteria, in a child.
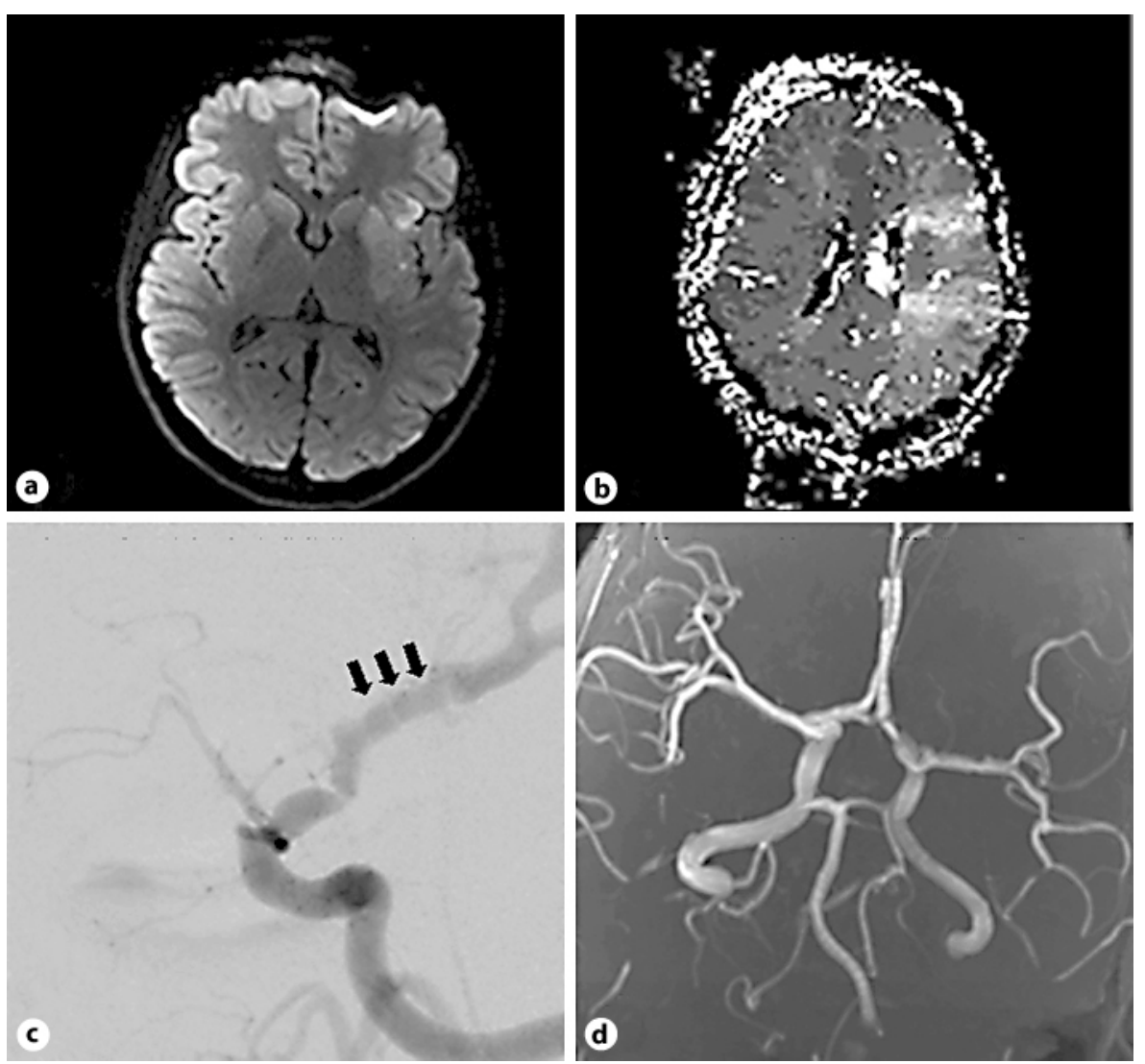

Fig. 1. a MRI with acute diffusion deficit in the left lentiform nucleus and the insula (diffusionweighted imaging). b MRI with hypoperfusion in the cortical left MCA territory (perfusion-weighted imaging). c Digital subtraction angiography of the left carotid artery with segmental stenosis (arrows) of the proximal MCA and distal ICA. d MRI-TOF (time of flight) angiography with irregular contrasting of the proximal left MCA.

\section{References}

1 Kirkham F, Sébire G, Steinlin M, Sträter R: Arterial ischaemic stroke in children. Review of the literature and strategies for future stroke studies. Thromb Haemost 2004;92:697-706.

2 The Atlantis ECASS and NINDS rt-PA Study Group Investigators: Association of outcome with early stroke treatment: pooled analysis of ATLANTIS, ECASS and NINDS rt-PA stroke trials. Lancet 2004;363:768-774.

3 Hacke W, Kaste M, Bluhmki E, et al: Thromboylsis with alteplase 3 to $4.5 \mathrm{~h}$ after acute ischemic stroke. $\mathrm{N}$ Engl J Med 2008;359:1317-1329. 
4 Amlie-Lefond C, de Veber G, Chan AK, et al: Use of alteplase in childhood arterial ischaemic stroke: a multicentre, observational, cohort study. Lancet Neurol 2009;8:530-536.

5 Janjua N, Nasar A, Lynch JK, Qureshi AI: Thrombolysis for ischemic stroke in children: data from the nationwide inpatient sample. Stroke 2007;38:1850-1854.

6 Arnold M, Steinlin M, Baumann A, et al: Thrombolysis in childhood stroke: report of 2 cases and review of literature. Stroke 2009;40:801-807.

$>7$ Rafay MF, Pontigon AM, Chiang J, Adams M, Jarvis DA, Silver F, Macgregor D, Deveber GA: Delay to diagnosis in acute pediatric arterial ischemic stroke. Stroke 2009;40:58-64.

-8 Puri V, Riggs G: Case report of fibromuscular dysplasia presenting as stroke in a 16-year-old boy. J Child Neurol 1999;14:233-238.

-9 Zurin AA, Houkin K, Asano T, Ishikawa T, Abe H: Childhood ischemic stroke caused by fibromuscular dysplasia of the intracranial artery - case report. Neurol Med Chir 1997;37:542-545.

10 DiFazio M, Hinds SR 2nd, Depper M, Tom B, Davis R: Intracranial fibromuscular dysplasia in a six-year-old child: a rare cause of childhood stroke. J Child Neurol 2000;15:559-562.

$\checkmark 11$ Heiserman JE, Drayer BP, Keller PJ, Fram EK: MR angiography of cervical fibromuscular dysplasia. AJNR Am J Neuroradiol 1992;13:1454-1457.

12 Plouin PF, Perdu F, La Batide-Alanore A, Boutouyrie P, Gimenez-Roqueplo AP, Jeunemaitre X: Fibromuscular dysplasia. Orphanet J Rare Dis 2007;2:28.

13 Amlie-Lefond C, Sébire G, Fullerton HJ: Recent developments in childhood arterial ischemic stroke. Lancet Neurol 2008;7:425-435. 